\title{
QUALIDADE DE VIDA NO TRABALHO: UMA INVESTIGAÇÃO MULTIVARIADA ENTRE DOCENTES
}

\section{LIFE QUALITY AT WORK: A MULTIVARIATE RESEARCH AMONG TEACHERS}

\section{D0l: http://dx.doi.org/10.21714/raunp.v10i1.1782}

\section{Carlos André Corrêa de Mattos}

Doutor em Ciências Agrárias. Professor da Faculdade de Administração (FAAD) da Universidade Federal do Pará (UFPa).

E-mail: carlosacmattos@hotmail.com

\section{Vânia Braga Damascno}

Graduada no Bacharelado em Administração. Universidade Federal do Pará. E-mail: vaniabraga2011@life.com

\section{Merabe Carvalho Ferreira da Gama}

Mestre em Gestão Pública. Bibliotecária-documentalista da Universidade Federal Rural da Amazônia. E-mail:

merabecarvalho@yahoo.com.br

\section{Cássio dos Santos Simão}

Mestrando do Programa de Pós-graduação em Administração da Universidade da Amazônia. E-mail: cassiossimao@gmail.com

\section{Nilson Luiz Costa}

Doutor em Ciências Agrárias. Professor da Universidade Federal de Santa Maria. E-mail: ecnilson@msn.com

Envio em: Dezembro de 2017

Aceite em: Janeiro de 2018

\section{RESUMO}

A Qualidade de Vida no Trabalho (QVT) é uma temática fortemente relacionada com a humanização e o bem-estar no ambiente laboral. Compreendêla desperta interesse científico e organizacional desde a década de 1950. Desta forma, o objetivo deste artigo é analisar a QVT de docentes de uma Instituição Federal de Ensino Superior e assim criar uma taxonomia capaz de possibilitar planos de melhoria. A metodologia foi exploratória e descritiva com levantamento de corte transversal e amostra não probabilística de 100 entrevistados selecionados por acessibilidade. 0 tratamento dos dados foi quantitativo. Os resultados revelaram cinco fatores, que foram denominados como Relações Interpessoais (17,90\%), Jornada de trabalho (16,46\%), Reconhecimento (13,98\%), Independência (12,24\%) e Carreira (6,86\%). 0s docentes foram classificados em dois grupos, um com $48 \%$ dos entrevistados levemente insatisfeitos com a QVT e outro com $52 \%$ levemente satisfeitos. Realização pessoal, autonomia e segurança profissional foram os melhores elementos da relação laboral, já os salários e as instalações físicas, os piores. Os resultados recomendam aprimorar aspectos da relação laboral como forma de melhorar a avaliação e fortalecer aspectos positivos do ambiente de trabalho.

Palavras-chave: Trabalho docente. Ambiente de trabalho. Gestão de pessoas. Análise multivariada. 


\section{ABSTRACT}

Life Quality at Work (LQW) is a theme strongly related to humanization and well-being at the working environment. Understanding it has aroused scientific and organizational interest since the 1950s. In this way, the objective of this paper is to analyze the LQW of teachers of some Federal Institution of Higher Education and thus to create a taxonomy capable to design plans for improvement. The methodology was exploratory and descriptive with a cross-sectional survey and non-probabilistic sample of 100 interviewees selected by accessibility. Data treatment was quantitative. Results showed five factors, namely Interpersonal Relations (17.90\%), Working Day (16.46\%), Recognition (13.98\%), Independence (12.24\%), and Career (6.86\%). Teachers were classified into two groups, one with $48 \%$ respondents slightly dissatisfied with the LQW and another with $52 \%$ slightly satisfied. Personal fulfillment, autonomy, and professional security were the best elements of the labor relationship, while wages and physical facilities were the worst. Results suggest improving aspects of the employment relationship as a way to improve the evaluation and strengthen positive aspects of the working environment.

Keywords: Teaching work. Working environment. People management. Multivariate analysis. 


\section{INTRODUÇÃO}

A profissão docente é reconhecida desde a década de 1980 como uma atividade com elevado risco à saúde (ORGANIZAÇÃO INTERNACIONAL DO TRABALHO, 1981). Na perspectiva organizacional, o adoecimento do docente agrava problemas como absenteísmo, produtividade, eleva o turnover, prejudica a qualidade do ensino, entre outros. Os efeitos dessas situações são evidentes, havendo necessidade de substituir os docentes, seja por contratações ou remanejamentos. Nesse contexto, o adoecimento docente, em muitos casos, é reflexo de problemas organizacionais sistêmicos que repercutem tanto na gestão das instituições de ensino, quanto na vida pessoal desses profissionais. Assim, seja pelas características da profissão, seja pela complexidade das organizações educacionais e sua importância para a sociedade moderna, diversos estudos têm se concentrado em compreender melhor as condições de trabalho desses profissionais.

Diehl e Marin (2016) reforçam o interesse científico sobre a saúde e as condições de trabalho dos docentes e complementam que as investigações sobre a temática assumiram características multidisciplinares. Desta forma, em face das diferentes perspectivas dos estudos sobre a profissão, destacam-se aqueles com foco na Qualidade de Vida no Trabalho (QVT). Pizio e Klein (2015), ao estudarem a temática sob a perspectiva de uma universidade federal brasileira, colocaram em evidência as mudanças ocorridas na profissão especialmente a partir do início desse século, quando foram observados aumentos expressivos nos investimentos na educação superior no Brasil e ocorreu a reestruturação produtiva da atividade docente. Assim, apesar de muito pesquisada, a QVT continua em evidência em estudos contemporâneos (ALFENAS; RUIZ, 2015; PIZIO; KLEIN, 2015; HIPÓLITO et al., 2017). Contudo, mesmo considerando o interesse científico, não há uma conceituação pacífica entre pesquisadores para o que vem a ser QVT, a não ser sua relação com a humanização no ambiente de trabalho (ALFENAS; RUIZ, 2015) e suas características de multidimensionalidade (SERINKAN; KAYMAKÇI, 2013).

Tongo (2015), ao referir-se à conceituação de QVT, destaca que existem muitas transposições da QVT com outros construtos de gestão de pessoas, como satisfação no trabalho e qualidade no trabalho. Para o autor, a falta de uma delimitação clara para a expressão é prejudicial para a compreensão do que represen- ta a QVT atualmente. Assim, eventuais incorreções, ou até mesmo, a utilização equivocada dos conceitos, muitos deles úteis para outros estudos, limitam o entendimento do que seja a QVT de fato. Isso se justifica porque muitos conceitos relacionados ao trabalho são, em grande parte, consequência da QVT, que, por sua vez, tem um espectro amplo e deve ser entendida em uma dimensão socioeconômica maior. Tongo (2015) argumenta que a QVT não pode ser extraída da concepção mais geral de qualidade de vida por ser um dos aspectos que formam da qualidade de vida. Assim, ambas fazem parte de um mesmo constructo, sendo a dimensão profissional inserida na perspectiva mais geral.

Desta forma, considerando as condições de trabalho dos docentes de universidades federais brasileiras e a relevância dessas instituições para o desenvolvimento econômico nacional, esse estudo assumiu como objetivo analisar as características da QVT, segundo as avaliações dos docentes da Universidade Federal do Pará (UFPA), concentrando-se, para tal, em responder ao questionamento: quais fatores influenciam na qualidade de vida no trabalho dos docentes e como esses profissionais podem ser classificados com relação a esses fatores? A UFPA é uma universidade da Região Norte do Brasil, formada por 14 institutos, 12 campi, 33 bibliotecas, 6 núcleos, 52 polos, uma escola de aplicação e dois hospitais universitários, foi criada em 1957 e reúne no campus da capital 40.275 estudantes e 2.254 docentes (UNIVERSIDADE FEDERAL DO PARÁ, 2016), estando posicionada entre as maiores universidades federais do país, mostrou-se como um local privilegiado para a investigação proposta neste estudo.

\section{FUNDAMENTAÇÃO TEÓRICA}

\subsection{QUALIDADE DE VIDA: CONCEITUAÇÃO, ORIGEM E EVOLUÇÃO}

Qualidade de Vida (QV) pode ser conceituada sob diferentes perspectivas teóricas segundo a área do conhecimento que se dedica a compreendê-la, sendo possível encontrar conceituações relacionadas à saúde (SCHMIDT et al., 2005), felicidade e satisfação pessoal (SRIVASTAVA; KANPUR, 2014), estilo de vida (NAHAS, 2003), entre outras. Entretanto, independentemente da abordagem teórica e conceitual escolhida, existem alguns aspectos comuns e mais gerais como a renda e a satisfação com aspectos valo- 
rizados na vida. Nesse contexto, a QV é uma temática abstrata com elevada complexidade e que exige delimitação para sua análise e compreensão (SOUZA et al., 2015).

Kluthcovsky e Takayamagui (2007) destacam que a expressão QV foi utilizada pela primeira vez por Pigou (1920), na obra "The economics of welfare". Contudo, ela somente ganhou repercussão ao ser utilizada pelo presidente dos Estados Unidos Lyndom Johnson, em 1964, ao afirmar que os objetivos dos bancos deveriam ser medidos não pelos balanços, mas pela qualidade de vida que proporcionavam às pessoas. Ao longo dos anos, a expressão QV foi intensamente utilizada por diversas áreas do conhecimento, aspecto que contribuiu para torná-la ainda mais subjetiva e com contornos pouco definidos. O tema despertou a atenção de diferentes pesquisadores, como sociólogos, filósofos, psicólogos, políticos, entre outros, em áreas como a saúde, urbanismo, padrões e condições de vida, entre outras, que foram investigadas com as mais variadas abordagens possíveis (REIS JUNIOR, 2008).

As pesquisas seminais sobre QV ocorreram nos Estados Unidos, inicialmente apenas com aspectos econômicos. Contudo, as mudanças na economia norte-americana, que progressivamente aumentou em complexidade e revelou graves problemas sociais, como a violência e criminalidade, fizeram com que aspectos sociais fossem inseridos na mensuração da QV (DAY; JANKEY, 1996). A diversidade de estudos e a necessidade de estabelecer delimitações para representar o construto, promoveram tamanha diversidade de conceituações que a QV não pode mais ser compreendida apenas sob umas poucas perspectivas teóricas (WHOQOL GROUP, 1998; LIMONGI-FRANÇA, 2004).

Mattos e Santana (2014), ao discorrerem sobre o tema, destacam que, a partir da década de 1990, as investigações fundamentadas na $\mathrm{QV}$ receberam aportes que contribuíram para seu amadurecimento teórico e metodológico, que passou a se pautar essencialmente na perceptiva da subjetividade e da multidimensionalidade. Na década de 1990, sintetizando diversas concepções sobre QV da época, a Organização Mundial da Saúde (ORGANIZAÇÃO MUNDIAL DA SAÚDE, 1998) passou a conceituar QV como a percepção que os indivíduos têm da própria vida, segundo um contexto cultural e um sistema de valores que resultam de seus objetivos, expectativas, padrões e preocupações com a vida.

Pereira (2006) já reforçava a subjetividade da QV ao afirmar que ela é essencialmente individual, uma vez que denota o bem-estar físico, emocional e a percepção de vida de cada um, aspectos diretamente influenciados por fatores externos ao indivíduo, especialmente aos fatores relativos a condições econômicas, sociais, culturais, ambientais, éticas e de estilo de vida. Assim, a QV assume uma dimensão pessoal e, portanto, variada, sendo subordinada às expectativas, projetos de vida e oportunidades percebidas no âmbito pessoal.

Para Minayo, Hartz e Buss (2000), a QV deve ser analisada em uma perspectiva ampla ao ser compreendida sob duas dimensões: uma delas, a representação social, construída com base em parâmetros subjetivos do ser humano como bem-estar, felicidade, amor, prazer e realização pessoal, e outra dimensão de características objetivas, pautadas na forma como o indivíduo satisfaz suas necessidades, sejam elas básicas ou secundárias, em conformidade com seu poder econômico e as características do local onde vive. Compreender a QV nessas perspectivas assume um conceito amplo que ultrapassa aspectos médicos e de saúde, apesar de esses elementos serem naturalmente indissociáveis da QV (TABELEÃO; TOMASI; NEVES, 2011).

\subsection{QUALIDADE DE VIDA NO TRABALHO: ASPECTOS CONCEITUAIS E EM INSTITUIÇÕES DE ENSINO SUPERIOR}

Notoriamente o homem sempre buscou melhorar suas condições de trabalho a fim de lutar pela sobrevivência (WALTON, 1973). A origem dessa busca é remota. Rodrigues (2008) destaca que os estudos de geometria de Euclides, 287 anos a.C., possibilitaram que os agricultores das margens do Rio Nilo melhorassem as práticas agrícolas. Da mesma forma, os estudos de Arquimedes sobre alavancas, ao facilitar mover objetos pesados com menor esforço, aliviaram os esforços dos trabalhadores e melhoraram sua produtividade. Logicamente, mesmo que a intenção desses matemáticos não fosse melhorar as condições de trabalho, seus estudos propiciaram avanços nessa área.

A Qualidade de Vida no Trabalho (QVT) deriva da compreensão mais geral de QV (SOUZA et al., 2015; TONGO, 2015). Estudos sistemáticos sobre a temática tiveram início nos primórdios da década de 1950, motivados pela preocupação com o bem-estar e a satisfação dos trabalhadores. Nesse contexto, preocupados em melhorar a produtividade, um grupo de 
pesquisadores investigou evidências dos efeitos do bem-estar e da satisfação com o trabalho na produtividade. Assim, movidos pelo interesse de integrar os indivíduos ao trabalho e, mais que isso, encontrar um alinhamento com os interesses dos trabalhadores e das organizações, uma equipe de pesquisadores londrinos, no Tavistock Institute, iniciaram estudos pautados na abordagem sociotécnica do trabalho. Essa abordagem compreende que a organização e a maneira como as tarefas são realizadas exercem influência nos trabalhadores, mais precisamente em seus sentimentos de responsabilidade, realização, reconhecimento, entre outros, materializando-se na satisfação com o trabalho (BIAZZI JR, 1994). Ao longo dos estudos, os pesquisadores londrinos utilizaram a expressão Qualidade de Vida no Trabalho (QVT), replicada por diversas pesquisas nos anos que se seguiram (FERNANDES; GUTIERREZ, 1998). No Brasil, os estudos de QVT foram iniciados aproximadamente 40 anos mais tarde, na década de 1990 (ZWIELEWSKI; TOLFO, 2016).

Limongi-França (2004), ao referir-se à QVT, a conceitua como uma prática baseada em um conjunto de ações desenvolvidas pelas organizações, com a finalidade de proporcionar melhorias e inovações gerenciais, tecnológicas e estruturais, que contribuam para o aperfeiçoamento do ambiente de trabalho. As ações de QVT culminariam com a negação dos métodos tayloristas de produção, pois têm como características principais a humanização do trabalho e a crença de que o rendimento dos trabalhadores aumenta na medida em que melhoram o bem-estar e a integração ao ambiente organizacional em uma dimensão biopsicosocial.

As preocupações com a QVT se justificam pela abrangência do tema tanto sob a perspectiva profissional, quanto organizacional e social, uma vez que seus reflexos se estendem amplamente ao ambiente de trabalho (FERNANDES; GUTIERREZ, 1998) incluindo insatisfação com o trabalho, aumento de conflitos internos, mau atendimento dos clientes, entre outros. Desta forma, as ações que se concentram na QVT são de interesse tanto dos trabalhadores, quanto de organizações, de pesquisadores (VELOSO; BOSQUETTI; LIMONGI-FRANÇA, 2005) e da sociedade em geral, pois, como destaca Guest (2002), a evolução do trabalho acabou por dominar a vida pessoal dos trabalhadores.

A QVT de docentes em instituições de ensino também desperta forte interesse dos estudos organi- zacionais, tanto de pesquisadores nacionais (KOETZ; REMPEL; PÉRICO, 2013: PIZZIO; KLEIN, 2015; SOUZA et al., 2015; LIMA; LIMA-FILHO, 2009), quanto internacionais (HART, 1994; JI-YOUNG AN; YOUNG-HEE YON; RUGGIERO, 2010; JAIN; THOMAS, 2016). Hart (1994), já pesquisava as experiências de QVT em instituições de ensino e em investigação com 1539 docentes australianos, constatou que o sofrimento psíquico e o moral contribuem igualmente para a QVT. Essa descoberta aumentou ainda mais os desafios de programas de QVT, por envolver aspectos complexos e ao considerar as limitações na capacidade de intervenção organizacional.

Complementarmente, ao investigar um hospital universitário na Coera, Ji-Youg An, Young-Hee e Ruggiero (2011) encontraram evidências de correlações significativas entre cultura organizacional, QVT e eficácia organizacional. Assim, ao colocarem em perspectiva esses aspectos, os autores constataram que a cultura organizacional exercia forte influência na QVT, que, por sua vez, se refletia na eficácia organizacional. Desta forma, para que ocorressem melhorias nos serviços prestados aos pacientes, havia necessidade de uma intervenção mais profunda na forma de gestão do hospital onde foi desenvolvido o estudo.

Nayeem e Triphathy (2012) investigaram docentes de cursos técnicos na Índia para procurar evidências que caracterizassem conflitos na relação entre a vida particular e o trabalho docente. Seus achados ratificaram a correlação positiva entre as condições de trabalho e a satisfação profissional, constatação indiferente para homens e mulheres. As conclusões da pesquisa confirmaram estatisticamente que havia necessidade de equilíbrio na relação entre a vida pessoal dos docentes e suas relações profissionais, uma vez que longas jornadas de trabalho estão entre os principais preditores de conflitos na relação família e trabalho. No Brasil, Koetz, Rempel e Périco (2013) alertam para a extensão e a elevada intensidade na jornada de trabalho de docentes, aspectos que limitam tanto o convívio social, quanto as atividades de interesses pessoais dos docentes. Os mesmos autores alertam para a necessidade de equilibrar os vários interesses que envolvem a profissão para reduzir as frustrações e a fadiga entre docentes. O estudo relata que a maior jornada de trabalho reduz fortemente os índices de QV.

Os aspectos de investigação da QVT são amplos nas instituições de ensino e na rotina dos docentes. Souza et al. (2015) destacam que a QVT de docentes 
é influenciada por grande número de aspectos como número de estudantes por turma, tarefas repetitivas, valorização do trabalho, feedback de superiores hierárquicos, autonomia na tomada de decisões, possibilidade de acesso a oportunidades profissionais de forma justa e igualitária, remuneração adequada ao trabalho realizado, possibilidades de integração social no ambiente de trabalho e uma relação de equilíbrio entre o trabalho e outros aspectos da vida pessoal. Os autores concluem que melhorar a percepção da QVT se reflete positivamente na qualidade dos serviços prestados e na produtividade dos docentes, proporcionando ganhos organizacionais expressivos.

Contudo, mesmo ao considerar a necessidade de as organizações promoverem ações que melhorem a QVT, as pesquisas (LIMA; LIMA-FILHO, 2009; PIOVEZAN; DAL RI, 2016) revelam um processo de precarização das condições de trabalho docente, aspecto que vem comprometendo a saúde e a relação ensino-aprendizagem, já que os docentes são os principais mediadores da construção do conhecimento e, com sua prática pedagógica, devem superar o ensino exclusivamente técnico e, sustentado na ética, incentivar uma prática crítica e transformadora nos estudantes (FREITAS et al., 2016).

\section{METODOLOGIA}

O método utilizado neste estudo classifica-se, segundo Creswell (2010), como exploratório e descritivo, com levantamento de corte transversal. A investigação foi feita na Universidade Federal do Pará (UFPA), no campus de Belém do Pará, Brasil. $O$ local da investigação foi selecionado por sua capacidade para fornecer informações sobre a qualidade de vida no trabalho de docentes em instituições públicas federais de ensino superior (IFES) e, assim, atender ao objetivo da investigação. A amostra foi não probabilística por acessibilidade. Nessa técnica, os entrevistados são escolhidos pela disponibilidade em participar do levantamento (CRESWELL, 2010; GIL, 2014). As amostras não probabilísticas limitam os resultados à amostra estudada, não podendo seus resultados ser estendidos à população. Neste estudo, os elos que uniram a população e, assim, possibilitaram identificar os integrantes da amostra foram: (1) ser docente e (2) trabalhar Universidade Federal do Pará e (3) estar lotado no campus de Belém do Pará. A amostra ocorreu em fase única, portanto, sem necessidade de etapas anteriores para sua identificação (CRESWELL, 2014).
No campus local da pesquisa, trabalham 2.254 (UFPA, 2016) docentes, que formaram o universo de pesquisa. Ao final do levantamento, foram obtidas 100 entrevistas válidas, que formaram o total da amostra estudada. As entrevistas foram feitas com a utilização de um questionário formado por duas seções. A primeira reuniu informações sociodemográficas e incluiu perguntas como idade, escolaridade, renda, tempo de serviço, motivos para escolher a carreira, satisfação com o trabalho, entre outras. As perguntas utilizadas nessa seção foram formuladas com respostas categóricas. A segunda seção foi adaptada de Pereira (2006) e contou com 19 variáveis, todas formuladas na forma afirmativa e com respostas em escala de Lickert. Essas variáveis foram relacionadas ao cotidiano do trabalho docente e tiveram a finalidade de contribuir para a compreensão dos aspectos relacionados com a QVT. A escala de Likert teve sete opções de respostas, que variaram de 1 para completamente insatisfeito, até 7 para completamente satisfeito. Os questionários foram antecedidos do Termo de Consentimento Livre e Esclarecido (TCLE), conforme padrões de ética em pesquisas com seres humanos.

O tratamento de dados foi quantitativo e utilizou estatística descritiva, multivariada e inferencial. As técnicas de estatística descritivas contribuíram para a compreensão ampla e inicial do fenômeno e incluíram a distribuição de frequência e o cálculo da média, mediana moda e desvio padrão. As técnicas multivariadas foram selecionadas entre as técnicas de interdependência, sendo escolhidas a análise fatorial exploratória (AFE) e a análise de agrupamentos (AA), por sua adequação ao objetivo da investigação.

A análise fatorial exploratória (AFE) é uma técnica multivariada, que tem como principal característica sua especial capacidade para sumarizar grande volume de dados em reduzido número de dimensões latentes, denominadas fatores. Os fatores que resultam da aplicação da técnica dificilmente seriam identificados com a utilização de outros procedimentos de análise de dados e podem substituir as variáveis originais, com a menor perda de informação possível (HAIR et al., 2009; FÁVERO et al., 2009).

Na pesquisa, tanto os fatores, como o questionário tiveram a confiabilidade verificada pelo coeficiente alpha de Cronbach. Após a etapa da análise fatorial exploratória (AFE) e verificada a consistência interna dos fatores, foi aplicada a análise de agrupamentos (AA). Esse procedimento se concentrou nas observações e teve por finalidade criar uma classificação, 
com base em um conjunto de médias de similaridade e de um algoritmo de aglomeração, que permitisse reunir os entrevistados em grupos com elevada similaridade entre seus integrantes e, ao mesmo tempo, mostrasse a maior diferença possível entre os grupos formados (HAIR et al., 2009; FÁVERO, 2009). Neste estudo, foi utilizada análise de agrupamentos hierárquica na forma aglomerativa, selecionada para reunir os docentes e assim identificar como eles avaliavam os aspectos da QVT. Esse procedimento, além de possibilitar quantificar os grupos, permitiu caracterizá-los criando uma taxonomia. Depois de formados os grupos, foram testados com o Teste $\mathrm{T}$ para amostras independentes para identificar: (1) os fatores que exerceram maior influência na formação dos grupos e (2) auferir se os grupos estavam corretamente classificados.

\section{ANÁLISE DE RESULTADOS}

Os docentes que participaram da pesquisa foram na maioria homens $(60 \%)$, casados $(60 \%)$, pais de até dois filhos (47\%), com idades entre 31 e 50 anos (66\%). Eles mostram escolaridade elevada, formada predominantemente por doutores $(48 \%)$, que concluíram o curso de graduação há mais de 16 anos (61\%) e tinham mais de 10 anos (62\%) de experiência docente. Trabalhando em regime de dedicação exclusiva (84\%), os entrevistados eram lotados em nove unidades administrativas da instituição, com maior concentração no Instituto de Ciências Sociais Aplicadas (54\%). A maior parte dos docentes escolheu a carreira docente depois de formados (59\%), seja por perceberem uma oportunidade promissora (33\%), seja pela vocação para ensinar (33\%). Destaca-se que a maioria $(80 \%)$ dos docentes entrevistados reconhece que a carreira atendeu a suas expectativas.

A análise descritiva dos aspectos da QVT, feita com base na média e no desvio padrão, possibilitou identificar que os docentes consideram entre os aspectos que contribuem favoravelmente para a QVT, a realização pessoal com o trabalho que realizam $(5,28 \pm 1,10)$, aspecto gratificante da carreira docente. Em seguida, ainda entre os aspectos favoráveis do local de trabalho, posicionaram-se a segurança e a estabilidade no emprego $(5,28 \pm 1,13)$. Nesse sentido, a estabilidade tem se destacado com frequência entre os principais atrativos da carreira pública, com evidencias confirmatórias em diversos estudos (ALMEIDA; MEIRELES, 2015; MATTOS et al., 2017).

Os docentes expressaram também de forma po- sitiva a autonomia com a qual realizam o trabalho $(5,23 \pm 1,13)$, o respeito à privacidade da vida pessoal $(5,09 \pm 1,16)$, a liberdade de expressão na instituição, tanto para discordar, quanto para sugerir $(5,23 \pm 1,13)$, a diversidade de relacionamentos profissionais no ambiente universitário $(4,91 \pm 1,17)$ e o tratamento sem preconceitos $(4,91 \pm 1,40)$ esses aspectos beneficiam a QVT.

Intermediariamente, com avaliação mais próxima da mediana da escala $(4,00)$, posicionaram-se questões relativas aos fluxos de informações nos processos de trabalho, que poderiam ser aperfeiçoados $(4,61 \pm 1,18)$, além da maior possibilidade de crescimento profissional $(4,61 \pm 1,24)$ e mais incentivos para a capacitação profissional $(4,33 \pm 1,41)$. Já com relação à jornada de trabalho $(4,16 \pm 1,42)$, destacaram-se o tempo disponível com familiares e amigos $(4,05 \pm 1,47)$ e o equilíbrio entre o tempo destinado ao trabalho e as necessidades pessoais e familiares $(3,95 \pm 1,49)$. Apesar de essas alternativas ainda estarem próximas da mediana da escala, sua proximidade dos estratos mais baixos, revelam aspectos que podem facilmente tornarem-se prejudiciais a QVT, recomendando atenção da instituição.

Quanto as condições declaradamente prejudiciais, observou-se a elevada quantidade de trabalho $(3,79 \pm 1,29)$, necessidade de jornadas complementares fora da instituição $(3,76 \pm 1,48)$, condições físicas do ambiente de trabalho, consideradas inadequadas $(3,72 \pm 1,45)$, e quantidade e adequação dos equipamentos necessários ao trabalho $(3,45 \pm 1,37)$. Esse conjunto de elementos deve ser observado pela instituição de forma prioritária, para proporcionar melhores condições de trabalho aos docentes, uma vez que, mostra aspectos considerados prejudiciais à QVT.

\subsection{ANÁLISE FATORIAL EXPLORATÓRIA}

Os testes de adequação à análise fatorial mostraram bom ajustamento, recomendando de forma satisfatória a utilização da técnica (Tabela 1). Desta forma, foram extraídos cinco fatores, que explicaram $70,44 \%$ da variância dos dados. A comunalidade foi adequada, posicionando-se acima de 0,537 para todas as variáveis, e as cargas fatoriais possibilitaram associar claramente as variáveis a um único fator. $\mathrm{O}$ coeficiente alpha de Cronbach revelou a elevada consistência interna, evidenciando resultados fidedignos, que ocorreram tanto para as variáveis em conjunto $(\alpha=0,900)$, quanto para os fatores individualmente 
(Fator $1=0,865$, Fator $2=0,843$, Fator $3=0,801$, Fator $4=0,784$ e Fator 5 $=0,795$ ).

Os cinco fatores reuniram 19 variáveis. Ao Fator 1, agregaram-se as variáveis V14, V15, V12, V19 e V16, esse fator foi denominado como Relações Interpessoais, pois captou as formas como os docentes se relacionam na organização. Ao Fator 2, associaram-se as variáveis V03, V05, V18, V17, V06, essa formação foi denominada de Jornada de Trabalho, uma vez que captou aspectos que exprimem a quantidade de tarefas em relação ao tempo necessário para realizá-las. Ao Fator 3, agregaram-se as variáveis
V01, V02, V07 e V03, esse conjunto foi identificado como Reconhecimento, pois reuniu as formas como o trabalho é recompensado e valorizado pela oferta de equipamentos necessários e de um ambiente de trabalho adequado. Ao Fator 4, reuniram-se as variáveis V08, V09 e V13, sendo o fator denominado como Independência, pois exprimiu a liberdade de ação e a segurança no exercício das tarefas cotidianas ao trabalho. Ao Fator 5, associaram-se as variáveis V10 e V11, esse fator foi denominado como Carreira, pelo fato da composição captar as possibilidades de progressão funcional e de qualificação profissional.

TABELA 1: RESULTADOS DA ANÁLISE FATORIAL.

\begin{tabular}{|c|c|c|c|c|c|c|c|}
\hline \multirow{2}{*}{ VARIÁVEIS } & & \multicolumn{5}{|c|}{ FATORES } & \multirow{2}{*}{$\mathbf{h}^{2}$} \\
\hline & & \multirow{2}{*}{$\begin{array}{c}1 \\
0,836\end{array}$} & \multirow{2}{*}{2} & \multirow{2}{*}{$\begin{array}{c}3 \\
0,180\end{array}$} & \multirow{2}{*}{4} & \multirow{2}{*}{$-0,021$} & \\
\hline $\begin{array}{l}\text { Relacionamento com os diversos profissionais da } \\
\text { instituição. }\end{array}$ & V14 & & & & & & 0,740 \\
\hline Respeito à privacidade na vida pessoal. & V15 & 0,717 & 0,198 & 0,138 & 0,401 & 0,216 & 0,780 \\
\hline Tratamento ausente de preconceitos. & V12 & 0,715 & 0,034 & 0,185 & 0,214 & 0,191 & 0,630 \\
\hline Realização pessoal pelo trabalho desenvolvido. & V19 & 0,690 & 0,185 & 0,152 & 0,078 & 0,193 & 0,577 \\
\hline Liberdade de manifestação, discordância e sugestão. & V16 & 0,623 & 0,072 & 0,194 & 0,434 & 0,283 & 0,699 \\
\hline Quantidade de atividades sob sua responsabilidade. & V03 & $-0,014$ & 0,793 & 0,219 & 0,097 & $-0,089$ & 0,694 \\
\hline Jornada de trabalho dentro da instituição. & V05 & $-0,076$ & 0,790 & 0,222 & 0,250 & $-0,049$ & 0,744 \\
\hline Tempo para lazer com a família e com os amigos. & V18 & 0,360 & 0,734 & 0,124 & $-0,119$ & 0,311 & 0,794 \\
\hline $\begin{array}{l}\text { Equilíbrio entre os horários de trabalho e as exigências } \\
\text { pessoais e familiares. }\end{array}$ & V17 & 0,392 & 0,730 & $-0,026$ & 0,099 & 0,174 & 0,727 \\
\hline Jornada de trabalho fora da instituição. & V06 & 0,072 & 0,650 & 0,182 & 0,141 & 0,236 & 0,537 \\
\hline $\begin{array}{l}\text { Salário justo quando comparado com as funções e } \\
\text { atividades que desempenha. }\end{array}$ & V01 & 0,171 & 0,231 & 0,772 & 0,097 & 0,092 & 0,696 \\
\hline $\begin{array}{l}\text { Salário justo quando comparado ao de outras } \\
\text { instituições. }\end{array}$ & V02 & 0,194 & 0,314 & 0,738 & 0,121 & $-0,134$ & 0,714 \\
\hline $\begin{array}{l}\text { Disponibilidade de equipamentos adequados para } \\
\text { desenvolver suas atividades. }\end{array}$ & V07 & 0,219 & 0,065 & 0,716 & 0,060 & 0,342 & 0,685 \\
\hline Condições ambientais de trabalho. & V03 & 0,099 & 0,091 & 0,699 & 0,162 & 0,165 & 0,694 \\
\hline Autonomia em relação ao trabalho desempenhado. & V08 & 0,259 & 0,133 & 0,183 & 0,762 & 0,084 & 0,706 \\
\hline Informações sobre o processo total de trabalho. & V09 & 0,088 & 0,233 & 0,309 & 0,747 & 0,144 & 0,737 \\
\hline Segurança e estabilidade no emprego. & V13 & 0,427 & 0,063 & $-0,089$ & 0,700 & 0,270 & 0,757 \\
\hline Possibilidade de crescimento profissional. & V10 & 0,207 & 0,065 & 0,262 & 0,304 & 0,796 & 0,842 \\
\hline $\begin{array}{l}\text { Incentivos e investimentos da instituição na } \\
\text { capacitação profissional }\end{array}$ & V11 & 0,306 & 0,236 & 0,120 & 0,167 & 0,758 & 0,766 \\
\hline Autovalores & & 3,40 & 3,13 & 2,65 & 2,32 & 1,84 & 13,34 \\
\hline Variância Explicada (\%) & & 17,90 & 16,46 & 13,98 & 12,24 & 6,86 & 70,44 \\
\hline Alpha de Cronbach & & 0,865 & 0,843 & 0,801 & 0,784 & 0,795 & 0,900 \\
\hline \multicolumn{8}{|c|}{$\begin{array}{l}\mathrm{KMO}=0,834 \text {, Teste de esfericidade de Bartllet }(\mathrm{X} 2)=1.030,30 \text {, significante a } \\
\text { Determinação do número de fatores pelo critério dos autovalores. } \\
\text { Extração dos fatores por ACP com rotação ortogonal pelo método Varimax. }\end{array}$} \\
\hline
\end{tabular}

Fonte: Pesquisa de campo (2017). 
O fator Relações Interpessoais, Fator 1, explicou $17,90 \%$ da variância e captou a valorização dos relacionamentos entre os diferentes profissionais que compartilham o ambiente de trabalho universitário. Fundamentado na realização pessoal, proporcionada pelo trabalho docente e pelo respeito às diferenças, à privacidade e à livre expressão, o fator refletiu a valorização dos relacionamentos livres de preconceitos, com participação e integração entre os servidores. Nesse contexto, o fator relacionou a necessidade de respeito, tanto com relação às diferenças individuais, quanto com relação à privacidade.

Aquino e Fernandes (2013) destacam que bons relacionamentos na organização são uma forma de atrair e manter os melhores profissionais. Salles e Frederight (2006) ampliam essa compreensão ao afirmarem que, quando as relações sociais melhoram na organização, melhora também a QVT. Em outra perspectiva, Morin (2001) identificou que, entre as principais razões que levam o ser humano ao trabalho, estão a necessidade de relacionamento com outras pessoas, a necessidade de construir e manter vínculos, a busca por uma ocupação, a necessidade de ter um objetivo na vida e para evitar a monotonia.

$\mathrm{O}$ fator Jornada de Trabalho, Fator 2, explicou $16,46 \%$ da variância e exprimiu a necessidade de promover um equilíbrio entre as diversas atividades realizadas pelos docentes. $\mathrm{O}$ fator sintetizou a relação entre a quantidade de tarefas e o tempo disponível e, mais que isso, o fator colocou em perspectiva a busca por uma harmonia entre as atividades profissionais $\mathrm{e}$ familiares. Sing (2010) destaca que o desequilíbrio na relação entre o trabalho e a vida privada leva a graves problemas de saúde, além da progressiva deterioração do desempenho profissional. Guest (2002) e Nayeem e Tripath (2012) alertam que, nas últimas décadas, ocorreu intensificação na quantidade e na complexidade no trabalho, situação que levou ao agravamento das relações trabalhistas, que passaram a ser vistas como desafios para o século XXI, tanto para a atuação dos departamentos de recursos humanos, quanto nas estratégias de organizações bem-sucedidas.

Ao concentrarem-se ao tema, Oliveira, Benatti e Alexandre (2006), em pesquisa com 91 médicos, encontraram riscos para a saúde física e psicológica entre aqueles que trabalhavam por mais de 40 horas semanais. Resultados semelhantes foram obtidos por Rocha e Sarriera (2006) ao estudarem a carreira docente, uma vez que os docentes são exigidos em dupla jornada de trabalho pela impossibilidade de realizar todas as tarefas no ambiente universitário. Os estudos de Rocha e Sarriera (2006) encontraram resultados significativos entre o aumento do número de horas de trabalho e a redução da saúde dos docentes. Assim, buscar um equilíbrio entre as diversas atividades realizadas pelos docentes é uma estratégia necessária para a manutenção e aprimoramento na QVT nas instituições de ensino.

O fator Reconhecimento, Fator 3, explicou 13,98\% da variância e captou as condições financeiras, materiais e físicas do ambiente de trabalho na instituição. Diversos estudos mostram os riscos da precarização do trabalho docente (LIMA; LIMA-FILHO, 2009; PIOVEZAN; DAL RI, 2016), especialmente quando investigam salários recebidos, exposição a agentes de risco, limitações de recursos materiais e humanos, que acabam por resultar em maior sobrecarga de trabalho para os docentes. Em comum, esses estudos percebem a perda de diretos conquistados ao longo dos anos, que colocam a carreira e as condições de trabalho em uma dimensão secundária perante as políticas públicas em geral.

Nesse sentido, ao investigar as condições físicas do local de trabalho na Universidade Federal do Mato Grosso do Sul (UFMS), Lima e Lima-Filho (2009) identificaram que, de maneira geral, havia falta de pessoal, materiais de consumo e equipamentos, todos considerados insuficientes para a realização das tarefas, situação agravada pela falta de apoio administrativo. Assim, as condições do local de trabalho e a remuneração recebida pelos docentes influenciam diretamente na QVT e, como tal, foram captadas nesse fator.

O fator Independência, Fator 4, explicou 12,24\% da variância e captou a liberdade de atuação docente ao reunir três elementos necessários ao exercício da profissão: (1) a autonomia no trabalho; (2) o acesso a informações; e (3) a segurança e estabilidade no emprego. Biroli (2016, p. 41), ao discorrer sobre o tema, alerta para a amplitude da expressão autonomia e suas diversas aplicações. Contudo, ele propõe uma conceituação no âmbito do indivíduo "[...] como aquele em que consciência e vontade atendem a motivações "próprias". Não se trata da suspensão do contexto das interações, mas da delimitação de um âmbito que deveria ser preservado para que a liberdade individual seja garantida".

Complementaram o fator a segurança e a estabilidade no emprego. Esses elementos, apesar de criticados no serviço público, por colocar todos os estáveis no mesmo nível, não distinguindo aqueles que se dedicam ao trabalho dos que não o fazem (BRESSER- 
-PEREIRA, 1996), evidenciam-se entre os aspectos que mais contribuem para a satisfação com o trabalho e para atrair profissionais para as carreiras públicas (MATTOS et al., 2017).

O fator Carreira, Fator 5, explicou $6,68 \%$ da variância dos dados e captou elementos que estimulam a qualificação profissional e a possibilidade de progressão na hierarquia organizacional, possibilitando, com isso, além de realização pessoal, maiores ganhos financeiros. $\mathrm{O}$ fator foi caracterizado essencialmente pelo desenvolvimento profissional e o aproveitamento da qualificação, seja na docência, seja na gestão universitária. $\mathrm{O}$ fator colocou em evidência a necessidade de fornecer estímulos que motivem a formação continuada especialmente pelo reconhecimento de mérito.

\subsection{ANÁLISE DE AGRUPAMENTOS}

A análise de agrupamento foi feita com base nos escores fatoriais calculados pelo método da regressão. Esse procedimento resultou em dois agrupamentos, um formado por 48 (48\%) docentes e outro por 52 (52\%). Os escores fatoriais, para toda a amostra, têm média zero $(0,00)$ e desvio padrão um $(1,00)$. Desta forma, para interpretá-los nos grupos, quanto mais os escores calculados para os agrupamentos de afas- tarem dos valores de referência $(0,00$ e 1,00$)$, maior será a contribuição do fator para a QVT se o sinal for positivo, ou prejudicial se o sinal for negativo. Completam a interpretação os valores dos desvios padrão, que mostram se os resultados dos grupos são mais ou menos dispersos do que a média geral.

A formação dos agrupamentos foi precedida da escolha de dois parâmetros. O primeiro é a forma de cálculo da distância geométrica entre as observações e o segundo, a forma como as observações serão reunidas. Assim, foram utilizados, respectivamente, a distância Euclidiana ao quadrado e o algoritmo de Ward. Ao término do processo de aglomeração, os grupos resultantes foram testados com o Teste $\mathrm{T}$ para amostra independente (Tabela 2). A escolha desse teste teve como finalidade verificar se os agrupamentos eram significativamente diferentes entre si e identificar os fatores que mais diferenciavam os grupos. Os resultados mostraram que o único fator que não apresentou diferenças significativas entre os grupos foi o Fator 1 (Relacionamento Pessoal) e que o Fator 3 (Reconhecimento) foi o que mais diferenciou os agrupamentos $(\mathrm{T}=-7,271, \mathrm{p}$-valor $=0,000)$. Os resultados gerais do teste permitem afirmar, com $95 \%$ de certeza, que os grupos eram diferentes para quatro dos cinco fatores identificados, estando corretamente classificados com relação a esses fatores.

TABELA 2: TESTE T PARA AMOSTRAS INDEPENDENTES DOS AGRUPAMENTOS.

\begin{tabular}{|l|c|c|c|c|}
\multirow{2}{*}{ FATORES } & \multicolumn{2}{|c|}{ TESTE F } & \multicolumn{2}{c|}{ TESTE T } \\
\cline { 2 - 5 } & VALOR & P-VALOR & VALOR & P-VALOR \\
\hline F1 - Relacionamento Interpessoal & 11,195 & 0,001 & $-0,526$ & $0,601^{* * *}$ \\
F2 - Jornada de Trabalho & 1,442 & 0,233 & $-2,056$ & $0,042^{* *}$ \\
F3 - Reconhecimento & 2,268 & 0,135 & $-7,271$ & $0,000^{*}$ \\
F4 - Independência & 5,172 & 0,025 & 3,321 & $0,001^{*}$ \\
F5 - Carreira & 11,972 & 0,001 & $-2,647$ & $0,010^{*}$ \\
\hline
\end{tabular}

Notas: * Significativo a $1 \% ;{ }^{* *}$ Significativo a $5 \% ;{ }^{* * *}$ Não significativo.

Fonte: Pesquisa de campo (2017).

O agrupamento 1 (Figura 1), foi formado por 48 $(48 \%)$ docentes, que percebem a maioria dos fatores como insatisfatórios na universidade. Eles manifestaram indiferença quanto aos Relacionamentos Interpessoais $(-0,056 \pm 1,28)$ e avaliaram como levemente prejudiciais a Jornada de Trabalho $(-0,211 \pm 1,03)$ e a
Carreira $(-0,272 \pm 1,16)$. A pior avaliação para o grupo ocorreu no Reconhecimento $(-0,613 \pm 0,94)$, considerado moderadamente prejudicial. $\mathrm{O}$ único fator avaliado como benéfico para a QVT na instituição segundo esse grupo foi o Independência $(0,334 \pm 1,12)$, considerado levemente satisfatório. 
FIGURA 1 - CARACTERÍSTICAS DOS AGRUPAMENTOS.

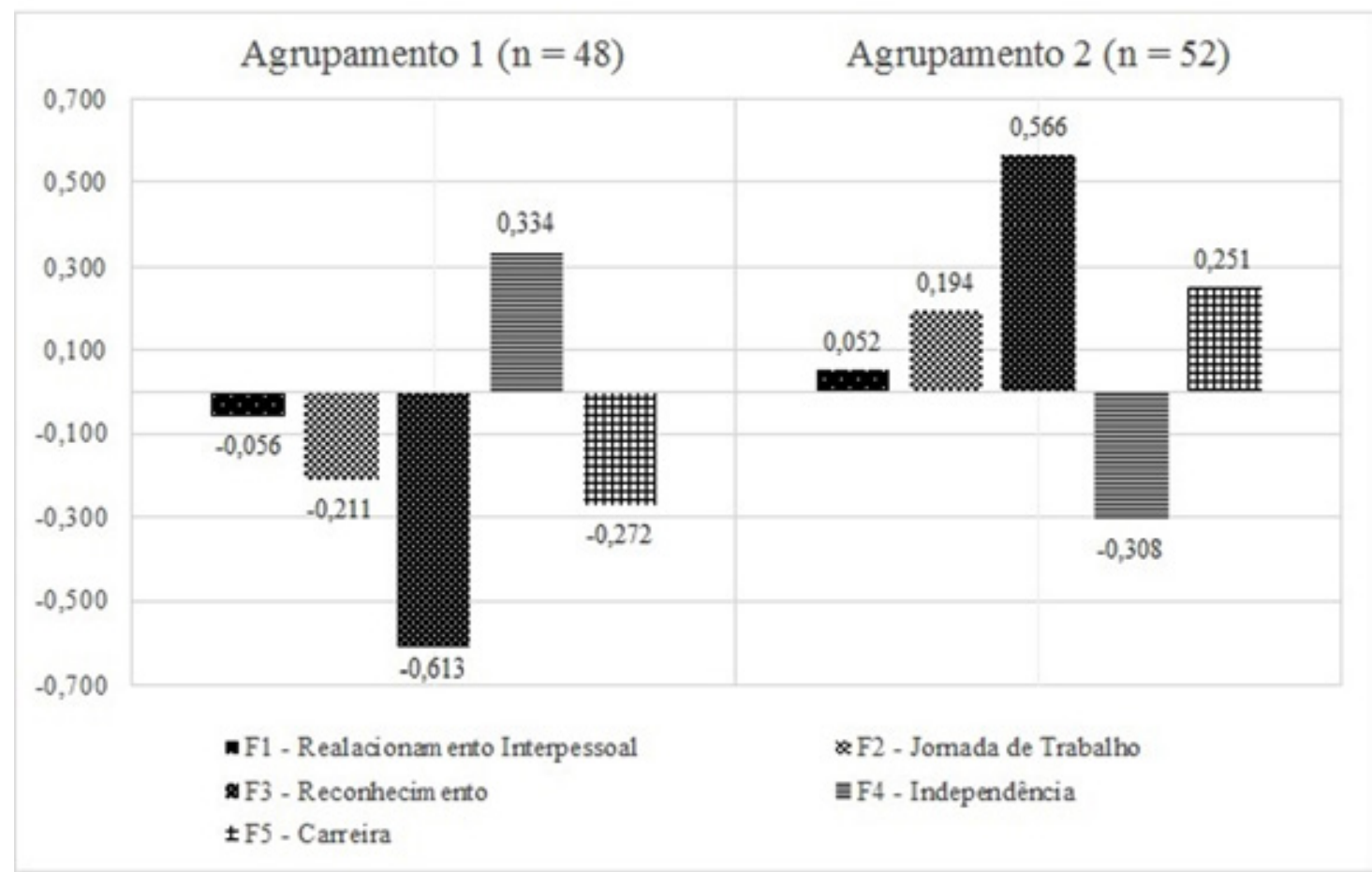

Notas: Pesquisa de campo (2017).

O agrupamento 2 foi formado por 52 (52\%) docentes e apresentou avaliação bem diferente do agrupamento 1 quanto à QVT, pois considerou favorável a maioria dos fatores investigados. A única semelhança entre os dois agrupamentos ocorreu no Relacionamento Interpessoal $(0,052 \pm 0,65)$, considerado indiferente por ambos e revelando um aspecto que merece a atenção da gestão da universidade. O agrupamento 2 percebe como levemente favoráveis para a QVT a Jornada de Trabalho $(0,194 \pm 0,94)$ e a Carreira $(0,251 \pm 0,75)$. Esse mesmo agrupamento avaliou o Reconhecimento $(0,566 \pm 0,67)$ como o fator que mais contribui para a QVT, mesmo considerando que ele tem intensidade moderada. A única avaliação levemente prejudicial foi quanto à Independência $(-0,308 \pm 0,77)$, insatisfatória, na opinião desse grupo.

Assim, de maneira geral, as avaliações dos docentes se dividiram. Destaca-se que os menores valores dos desvios padrão no agrupamento 2 mostram que as respostas estão mais concentradas em torno da média, o que lhe melhora a qualidade da avaliação e indica que esse grupo é mais conclusivo por ser mais coeso. Contudo, mesmo assim, os resultados recomendam o aprofundamento da investigação, especialmente pela limitação decorrente do processo de amostragem e reforçam a neces- sidade de ações voltadas para a QVT para melhorar a avaliação dos docentes.

\section{CONCLUSÃO}

Os docentes entrevistados eram, em sua maioria homens casados, com filhos, entre 31 e 50 anos, doutores e com mais de 10 anos de experiência na área docente. Observou-se que em relação à QVT no que se refere à realização com o trabalho desenvolvido, trabalho gratificante, segurança e estabilidade no emprego, foram obtidas as respostas mais favoráveis. As respostas intermediárias ocorreram em relação ao crescimento na carreira, incentivo à capacitação e até mesmo ao tempo despendido na Instituição, que não favorece a relação com a família, pontos estes que merecem ajustes por parte da Instituição.

Quanto aos aspectos que mostraram insatisfação por parte dos docentes, posicionaram-se a infraestrutura da universidade, o excesso de trabalho tanto internamente quanto fora da instituição, que, por serem os aspectos mais prejudiciais, devem ser objeto de maior interesse institucional e ajustes a fim de aumentar o nível de QVT dos docentes. A análise fatorial captou cinco fatores 
- Relação interpessoal, Jornada de trabalho, Reconhecimento, Independência e Carreira - e a análise de agrupamentos identificou dois grupos de docentes, praticamente com o mesmo quantitativo de integrantes, sendo um com avaliação levemen- te negativa da QVT e outro que manifestou avaliação contrária levemente positiva, ambos com baixa intensidade em suas avaliações, revelando um ambiente mediano em matéria de QVT na instituição pesquisada.

\section{REFERÊNCIAS}

ALFENAS, R. A. S; RUIZ, M. V. Um panorama sobre qualidade de vida no trabalho na administração pública brasileira no período de 2007 a 2013. Organizações em Contexto, São Paulo, v.11, n.22, p. 143-167, jul. /dez. 2015.

ALMEIDA, D. R.; MEIRELES, A. S. Satisfação e Trabalho: uma análise do grau de satisfação dos servidores em estágio probatório da UFBA. In: COLÓQUIO INTERNACIONAL DE GESTÃO UNIVERSITÁRIA, 15, 2015, Mar Del Plata. Anais..., Argentina, 2015

AQUINO, A. S.; FERNANDES, A. C. P. Qualidade de vida no trabalho. Journal of the Health Sciences Institute, São Paulo, v. 31, n. 1, p. 53-58, jan. /mar. 2013.

BIAZZI, JR, F. O trabalho e as organizações na perspectiva sociotécnica. Revista de Administração de Empresas, São Paulo, v.1, n. 34, p. 30-37, jan./fev. 1994.

BIROLI, F. Autonomia, preferências e assimetria de recursos. Revista Brasileira de Ciências Sociais, São Paulo, v. 30, n. 90, p. 39-56, fev. 2016.

BRESSER-PEREIRA, L. C. Da administração pública burocrática à gerencial. Revista do Serviço Público, Brasília, v. 47, n. 1, p. 1-28, jan. /abr. 1996.

CRESWELL, J. W. Projeto de pesquisa: métodos qualitativo, quantitativo e misto. Porto Alegre: Artmed, 2010.

DAY, H.; JANKEY, S.G. Lessons from the literature: toward a holistic model of quality of life. Thousand Oaks: Sage, 1996.

DIEHL, L.; MARIN, A.H. Adoecimento mental entre professores brasileiros: revisão sistemática da literatura. Estudos Interdisciplinares em Psicologia, Londrina, v. 7, n. 2, p. 64-85, dez. 2016.

FÁVERO, L. P. et al. Análise de dados: modelagem multivariada para tomada de decisões. São Paulo: Campus, 2009.

FERNANDES; E. C.; GUTIERREZ L. H. QVT - uma experiência brasileira. Revista de Administração, São Paulo, v. 23, n.4, p. 29-38, out. / dez. 1988.

FREITAS et al. Saberes docentes sobre processo ensino-aprendizagem e sua importância para a formação profissional em saúde. Interface Comunicação Saúde e Educação, Botucatu, n. 20, v. 57, p. 437-448, abr. / jun., 2016

GIL, A. C. Métodos e téenicas de pesquisa social. São Paulo: Atlas, 2014.

GUEST, D. E. Perspectives on the study of work-life balance. Social Science Information, v. 41, n. 2, p. 255 279 , jun. 2002. 
HAIR JR. et al. Análise multivariada de dados. Porto Alegre: Bookman, 2009.

HIPÓLITO et al. Quality of working life: assessment of intervention studies. Revista Brasileira de Enfermagem, Brasília, v. 70, n. 1, p. 178-86, 2017.

JAIN, Y.; THOMAS, R. A study on quality of work life among the employees of a leading pharmaceuticals limited company of Vadodara district. International Journal of Applied Research, Delhi, v. 2, n. 5, p. 926934, 2016.

JI-YOUNG, AN; YOUNG-HEE, YON; RUGGIERO, J. S. Organizational Culture, Quality of Work Life, and Organizational Effectiveness in Korean University Hospitals. Journal of Transcultural Nursing, Alabama, v. 22, n. 1, p. 22-30, jun. 2010.

KLUTHCOVISKY, A. C. G. C.; TACAYANAGUI, A. M. M. Qualidade de vida: aspectos conceituais. Revista Salus-Guarapuava, Paraná, v. 1, n. 1, jan. /jun, 2007.

KOETZ, L.; REMPEL, C.; PÉRICO, E. Qualidade de vida de professores de Instituições de Ensino Superior Comunitárias do Rio Grande do Sul. Revista Ciência \& Saúde Coletiva, Rio de Janeiro, v. 18, n. 4, p. 10191028, abr. 2013.

LIMA, M. F. E. M; LIMA-FILHO, D.O. Condições de trabalho e saúde do/a professor/a universitário/a. Ciências \& Cognição, Rio de Janeiro, v. 14, n. 3, p. 62-82, nov. 2009.

LIMONGI-FRANÇA, A. C. Qualidade de vida no trabalho: conceitos e práticas na sociedade pós-industrial. São Paulo; Atlas, 2004.

MATTOS, C.A.C.; SANTANA, A. C. As contribuições da pecuária leiteira para os agricultores familiares: um estudo no sudeste do Estado do Pará. Revista Extensão Rural, v. 21 n. 1, p. 56-72, jan. / mar. 2014.

MATTOS, C.A.C.; VIDAL, J. P.; LIRA, M. S.; COSTA, N. L.; ABUD, G. M. B. Satisfação e trabalho na administração pública federal: uma investigação entre servidores do ensino superior. Revista Cereus, v. 9, n. 1, p. 57-75, jan. / abr. 2017.

MINAYO, M. C. S. HARTZ, Z.M.A., BUSS, P.M. Qualidade de vida e saúde: um debate necessário. Revista Ciência e Saúde Coletiva, Rio de Janeiro, v. 5. n. 1, jan. /jun. 2000.

MORIN, E. M. Os sentidos do trabalho. Revista de Administração de Empresas, São Paulo, v. 41, n. 3, p. 8-19, jul. /set. 2001.

NAHAS, M.V. Atividade física, saúde e qualidade de vida: conceitos e sugestões para um estilo de vida ativo. 3. ed. Londrina: Midiograf, 2003.

NAYEEM, M. A.; TRIPATHY, M.R. Work-life balance among teacher of thechnical institutions. The Indian Journal of Industrial Relations, v.47, n. 4, p. 724-736, abr. 2012.

OLIVEIRA, A. P. B. M.; BENATTI, M. C. C.; ALEXANDRE, N. M. C. Condições de vida e trabalho de médicos em um hospital universitário. Revista Gaúcha de Enfermagem, Porto Alegre, v. 27, n. 1, p. 53-59, mar. 2006.

ORGANIZAÇÃO INTERNACIONAL DO TRABALHO. Empleo y Condiciones de trabajo del personal docente. Genebra, 1981.

ORGANIZAÇÃO MUNDIAL DA SAÚDE. Health promotion evaluation: recommendations to policymakers. 
Copenhagen: European Working Group on Health Promotion Evaluation, 1998.

PEREIRA, O. A. V. Qualidade de vida no trabalho de docentes universitários de uma Instituição pública e outra privada do leste de Minas Gerais. 2006. 99 f. Dissertação (Mestrado em Meio Ambiente e Sustentabilidade). Centro Universitário de Caratinga. Minas Gerais, 2006.

PIGOU, A.C. The economics of welfare. London: Macmillan, 1920.

PIOVEZAN, P. R.; DAL RI, N.M, A precarização do trabalho docente no estado de São Paulo: 20 anos de reformas. Educação Temática Digital, Campinas, v. 18, n. 1, p. 178-197, jan. / abr, 2016.

PIZIO, A.; KLEIN, K. Qualidade de vida no trabalho e adoecimento no cotidiano de docentes do ensino superior. Educação \& Sociedade, Campinas, v. 36, n. 131, p. 493-513, abr./ jun. 2015.

REIS JUNIOR, D. R. Qualidade de Vida no Trabalho: Construção e validação do questionário QWLQ-78. 2008. 114 f. Dissertação (Mestrado em Engenharia de Produção). Universidade Tecnológica Federal do Paraná. Ponta Grossa, 2008.

ROCHA, K.B.; SARRIERA, J.C. Saúde percebida em professores universitários: gênero, religião e condições de trabalho. Revista Semestral da Associação Brasileira e Psicologia Escolar e Educacional, Uberlândia, v. 10, n. 2, p. 187-196, jul. / dez. 2006.

RODRIGUES, M. V. Qualidade de vida no trabalho: evolução e análise do nível gerencial. Rio de Janeiro, Petrópolis: Vozes, 2008.

SALLES, P. E. M.; FEDERIGHI, W. J. Qualidade de vida no trabalho (QVT): a visão dos trabalhadores. O Mundo da Saúde, v. 30, n. 2, p. 263-278, abr./jun. 2006.

SCHMIDT, S et al. The conceptual relationship between health indicators and quality of life: results from the cross-cultural analysis of the EUROHIS field study. Clinical Psychology \& Psychotherapy, Hoboken, v.2, n.1, p.28-49, 2005.

SERINKAN, C.; KAYMAKÇI, K. Defining the Quality of Life Levels of the Nurses: a study in Pamukkale University. Procedia: Social and behavioral Sciences, New York, v. 89, p. 5820-584, 2013.

SING, A. A study on the perception of work-life balance policies among software professionals. The IUP Journal of Management Research, Virginia, v. 9, n. 2, p. 51-79, fev. 2010.

SOUZA, A. S. et al. Fatores associados à qualidade de vida no trabalho entre professores do ensino superior. Arq. Ciênc. Saúde. São José do Rio preto, v. 22, n. 4, p. 46-51, out-dez, 2015.

SRIVASTAVA, S.; KANPUR, R. A Study on Quality of Work Life: Key Elements \& It's Implications, Journal of Business and Management, Irvine, USA, v.16, n.3, p. 54-59, mar. 2014.

TABELEÃO, V. P.; TOMASI, E.; NEVES, S. F. Qualidade de vida e esgotamento profissional entre docentes da rede pública de Ensino Médio e Fundamental no Sul do Brasil. Cadernos de Saúde Pública, Rio de Janeiro, v. 27, n. 12, p. 2401-2408, dez, 2011.

TONGO, C. I. Social responsibility, quality of life and motivation to contribute in the Nigerian Society. Journal of Business Ethics, v. 126, n. 2, p. 219-233, jan. 2015.

UNIVERSIDADE FEDERAL DO PARÁ. Universidade Federal do Pará em números, 2016. Disponível em: <http://www.ufpanumeros.ufpa.br/>. Acesso em: 16 fev. 2017. 
VELOSO, H.M; BOSQUETTI, M.A.; LIMONGI-FRANÇA, A. C. A concepção gerencial dos programas de qualidade de vida no trabalho (QVT) no setor elétrico brasileiro. In: SEMINÁRIOS EM ADMINISTRAÇÃO, VII, 2005. São Paulo. Anais... São Paulo: FEA-USP, 2005.

WALTON, R. E. Quality of work life: what is this? Sloan Management Review, n. 15, v. 1, p. 11-21, dec. 1973

WHOQOL Group, The O desenvolvimento do WHOQOL-100, Porto Alegre, UFRGS, 1998.

ZWIELEWSKI, G.; TOLFO, S. R. Qualidade de vida no trabalho de profissionais expatriados para a Índia e China. Prevalência revelada e arbitragem espacial: determinando um ranking de qualidade de vida para as regiões metropolitanas do Brasil. Revista Eletrônica de Administração, Porto Alegre, n. 84, v. 2, p. 510-542, mai. / ago. 2016. 